\title{
Investigación en catálisis en la Universidad Michoacana
}

\author{
José Luis Rico Cerda*
}

RESUMEN: En este trabajo se hace una breve reseña de la investigación y actividades relacionadas con la catálisis llevadas a cabo en la Facultad de Ingeniería Química de la Universidad Michoacana de San Nicolás de Hidalgo. Se mencionan los proyectos y las tareas de investigación, la infraestructura disponible, así como las colaboraciones con diferentes instituciones. Finalmente, se mencionan los principales logros.

PALABRAS CLAVE: Catálisis, proyectos de investigación, infraestructura.

Abstract: A brief review of the research activities on catalysis at the Department of Chemical Engineering of the University of Michoacan is herein described. The approved research projects, the available infrastructure in the laboratory and the collaboration with other institutions are also mentioned. The main achievements are finally commented.

KEYWORDS: Catalysis, research projects, infrastructure.

\section{Introducción}

Las actividades de investigación en el área de catálisis comienzan en la Universidad Michoacana de San Nicolás de Hidalgo (UMSNH) con la incorporación del autor de esta reseña a la Facultad de Ingeniería Química (FIQ), perteneciente a esta institución, en 1993. Sin embargo, y debido a las dificultades encontradas en todo comienzo, fue hasta el año de 1996 que se realizó el primer trabajo de tesis relacionado con catálisis. Los primeros temas de investigación fueron sobre la síntesis de materiales y su caracterización. Cabe mencionar que gracias a los apoyos recibidos del Instituto de Investigaciones Metalúrgicas de la UMSNH, nuestras actividades de investigación fueron posibles y continúan siendo apoyadas en la actualidad. Esto se debe a que dicho instituto cuenta con infraestructura muy importante para nuestros fines, como son, por ejemplo, los equipos de microscopía electrónica, difracción de rayos X, espectroscopía de infrarrojo, entre otros. Asimismo, del 22 al 24 de noviembre de 1998, conjuntamente con la Academia de Catálisis A.C., organizamos por primera vez en nuestra institución, el IV Encuentro Nacional de Catálisis Heterogénea, lo cual vino a robustecer el desarrollo de esta área de investigación en la región occidente de nuestro país. Actualmente, somos

Recibido: 23 de enero de 2017. Aceptado: 17 de marzo de 2017.

* División de Estudios de Posgrado de la Facultad de Ingeniería Química, Universidad Michoacana de San Nicolás Hidalgo. Gral. Francisco J. Múgica S/N, Ciudad Universitaria, 58030, Morelia, Mich., México. Correspondencia: (jlrico@umich.mx). 


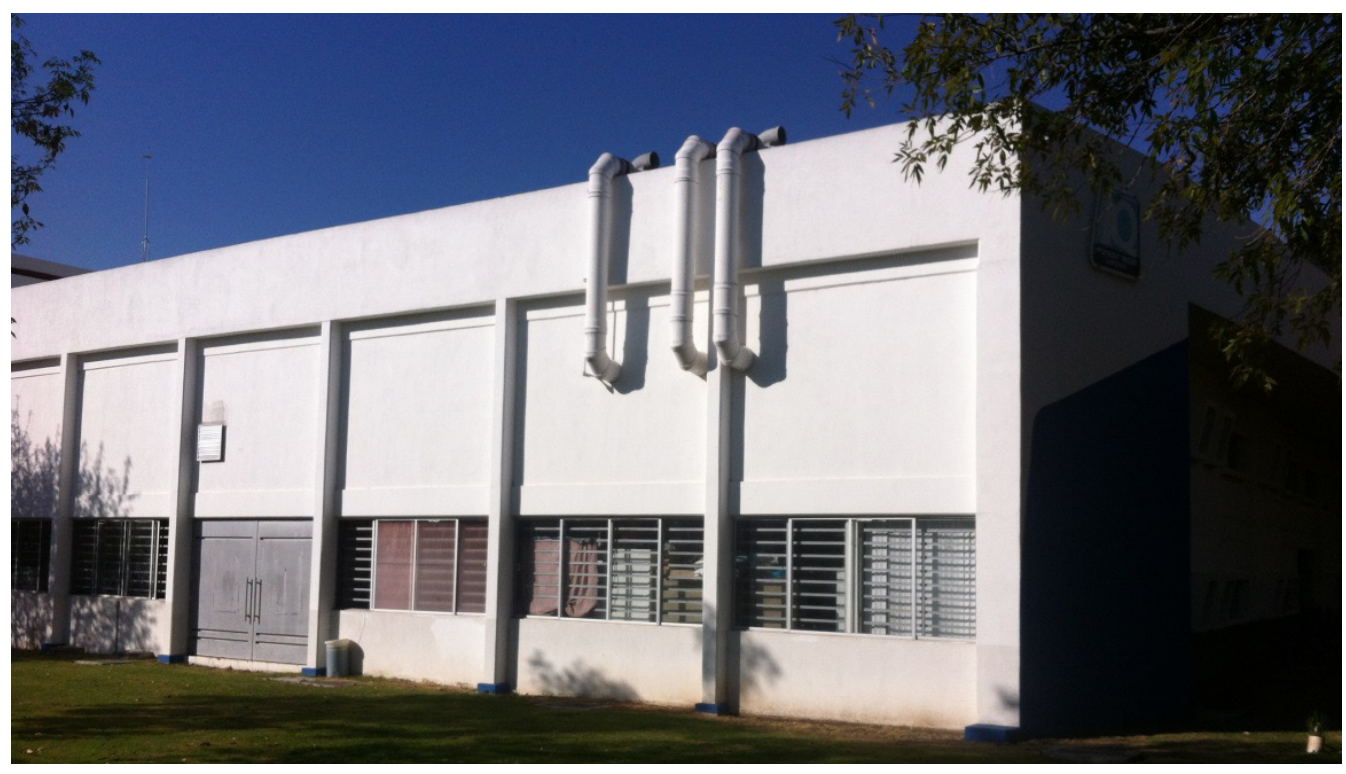

FIGURA 1. Edificio de posgrado de la FIQ de la UMSNH.

cinco profesores los que realizamos investigación en catálisis, y, con excepción de un profesor, todos nos formamos en el Laboratorio de Catálisis de la FIQ de la UMSNH: Dr. R. Huirache Acuña, Dr. J. Espino Valencia, Dr. M. Arroyo Albiter y el Dr. H. González Rodríguez. Además, hasta la fecha, 111 estudiantes han realizado su tesis y obtenido su grado en temas relacionados con la catálisis en nuestra institución.

\section{Líneas de investigación y perspectivas de desarrollo}

Las líneas de investigación relacionadas con la catálisis en las que trabajamos son: fuentes alternas de energía, hidrotratamiento, degradación de contaminantes por medio de la fotocatálisis, reformación del metano tradicional y en seco, síntesis del amoniaco, uso de materiales naturales y de desecho como catalizadores, hidrogenaciones e isomerizaciones. Las actividades incluyen síntesis, caracterización y prueba de catalizadores en reacciones químicas, las cuales se llevan a cabo en diferentes tipos de reactores. Los tipos de materiales catalíticos usados son: metales, óxidos, sulfuros, fosfuros, nitruros y carburos metálicos. Es de mencionarse que el potencial de aplicación de estos materiales es enorme, ya sea en el campo de la catálisis, como en el área de nuevos materiales nanométricos de morfologías diferentes.

Continuaremos la investigación sobre nuevos materiales catalíticos y su aplicación en diferentes reacciones químicas. Prevemos que la investigación en nuestro laboratorio para los próximos años se concentrará más en la sín- 
tesis de biocombustibles y problemas de contaminación del aire y agua. Seguiremos con el desarrollo de catalizadores para la obtención de hidrógeno, para la síntesis de 2,5-dimetilfurano como biocombustible obtenido a partir de la biomasa y en la obtención del biodiesel a partir de diferentes materias primas. Sobre problemas de contaminación del agua seguiremos con el desarrollo de fotocatalizadores, además, probaremos nuevas rutas combinadas fotocatalíticas-enzimáticas, para lo cual se colabora actualmente con el Dr. J. M. Sánchez Yáñez. También continuaremos con la síntesis de nanomateriales para su aplicación en diferentes campos. Por caso, actualmente desarrollamos materiales quitosano-óxido de fierro, interactuando con Dra. N. Flores Ramírez y el Dr. S. R. Vázquez García, en la obtención de nuevos materiales para reemplazo de hueso, por ejemplo, con el compuesto $\mathrm{Ti}_{6} \mathrm{Al}_{4} \mathrm{~V}$, investigación a cargo del Dr. L. R. Olmos Navarrete. Contamos además con la colaboración del Dr. P. Navarro Santos, quien realiza cálculos sobre química teórica y computacional. Además, seguiremos colaborando con expertos de instituciones mencionadas en el apartado sobre colaboración, lo cual fortalecerá aún más el desarrollo de esta área de investigación en nuestra institución.

\section{Proyectos}

Los proyectos apoyados hasta la fecha son:

- Catalizadores de Pt para la hidrogenación isomerativa de trans2-buteno.

- Hidrodesoxigenación del fenol con catalizadores trimetálicos.

- Desarrollo de catalizadores de hidrodesulfuración de $\mathrm{Co}(\mathrm{Ni}) \mathrm{MoW}$ soportados en sílices mesoporosas (HMS y SBA-16) modificadas con aluminio: efecto del soporte y del promotor.

- Desarrollo de catalizadores de hidrodesulfuración de $\mathrm{Co}(\mathrm{Ni}) \mathrm{MoW}$ soportados en sílices mesoporosas (HMS y SBA-16) modificadas con aluminio.

- Desarrollo de catalizadores trimetálicos de hidrodesulfurización soportados en sílices mesoporosas modificadas con Titanio.

- Síntesis de nanopartículas de sulfuros bimetálicos soportadas sobre nanotubos de carbono y su aplicación en hidrodesulfuración.

- Síntesis hidrotérmica de nanopartículas de sulfuros metálicos soportadas sobre nanotubos de carbono y su aplicación en hidrodesulfuración.

- Desarrollo de catalizadores a base de sulfuros y fosfuros de metales de transición y su aplicación en hidrodesulfuración.

- Caracterización de materiales catalíticos superácidos a base de titania.

- Reformación del metano en seco.

- Titania sulfatada en la reacción de isomerización del n-butano.

- Isomerización de n-butano en sólidos de gran acidez: efecto de las condiciones de reacción. 
- Nanomateriales de $\mathrm{MoS}_{2}$ como catalizadores.

- Conversión heterogénea de aceite a biodiesel mediante un proceso consecutivo de esterificación ácida/transesterificación básica.

- Síntesis y caracterización de titania mesoestructurada por el método EISA y su aplicación fotocatalítica: influencia del método de secado y precursor de titanio.

- Modificación de derivados de resina de pino para la elaboración de productos de especialidad.

- Biodiesel como fuente alterna de energía.

- Síntesis de biodiesel en presencia de catalizadores sólidos.

- Síntesis de biodiesel a partir de aceite de higuerilla y en presencia de catalizadores sólidos.

- Nitruro de CoMo modificado con paladio.

- Incorporación del níquel en la estructura del nitruro de CoMo.

- Síntesis de compuestos trimetálicos másicos Ni-W-Mo por el método hidrotérmico para hidrodesulfuración.

- Catalizadores trimetálicos NiWMo soportados y probados en reacciones de hidrotratamiento (hidrodesulfuración e hidrodesnitrogenación): modificación superficial del soporte óxido mixto aluminiotitanio con magnesio.

- Procesos de oxidación avanzados.

- Influencia del templante en la obtención de titania mesoestructurada y actividad fotocatalítica.

De estos proyectos, 21 (84 \%) fueron apoyados y financiados por la Coordinación de la Investigación Científica de la UMSNH; 2 proyectos (8 \%) por el CONACyT; 1 proyecto por la SEP-PROMEP, y otro por el COECyT, Michoacán.

\section{Colaboraciones}

A continuación se enlistan las universidades o instituciones con las que se ha tenido, y se tiene colaboración en actividades de investigación:

\section{Nacionales}

Benemérita Universidad Autónoma de Puebla.

Centro de Investigación y de Estudios Avanzados, Querétaro.

Instituto Potosino de Investigación Científica y Tecnológica, San Luis Potosí.

Instituto Tecnológico de Morelia.

Universidad Autónoma del Estado de México.

Universidad Autónoma de Querétaro.

Universidad Juárez Autónoma de Tabasco.

Universidad Autónoma Metropolitana Iztapalapa.

Universidad Autónoma de México: Centro de Nanociencias y Nanotec- 
nología; Facultad de Química; Centro de Física Aplicada y Tecnología Avanzada.

Universidad Autónoma de Sinaloa.

Universidad de Guanajuato.

\section{Extranjeras}

Catalysis Research Center, Hokkaido University, Japón.

Eidgenössische Technische Hochschule (ETH), Zürich, Suiza.

Facultad de Ingeniería Química, Universidad de Zaragoza, España.

Instituto de Catálisis y Petroleoquímica, Madrid, España.

Institut de Recherches sur la Catalyse et l'Environnement de Lyon, Francia.

School of Chemistry, University of Glasgow, Scotland, Reino Unido.

Slovenská Vysoká Škola Techniská vo Bratislave, Eslovaquia.

University of California Riverside, USA.

University of Liverpool, Reino Unido.

\section{Infraestructura}

La infraestructura con la que contamos es la siguiente: equipos para la reducción, oxidación y sulfuración; sistema para deposición química de vapor (CVD, por sus siglas en inglés); reactor para alta presión y temperatura; reactor tubular continuo; dos cromatógrafos de gases; sistema de adsorción acoplado a un equipo de infrarrojo con transformada de Fourier (FT-IR, por sus siglas en inglés); equipo para la determinación de áreas superficiales; equipo de oxidación-reducción; reactores tipo batch para alta presión y temperatura; equipo de análisis térmico simultaneo (TGA-DSC, por sus siglas en inglés) acoplado a espectrómetro de masas; equipo de ultrasonido para reacciones químicas.

FIGURA 2. Sistemas de adsorción para medición de áreas superficiales y equipo de reacción para alta presión y temperatura.
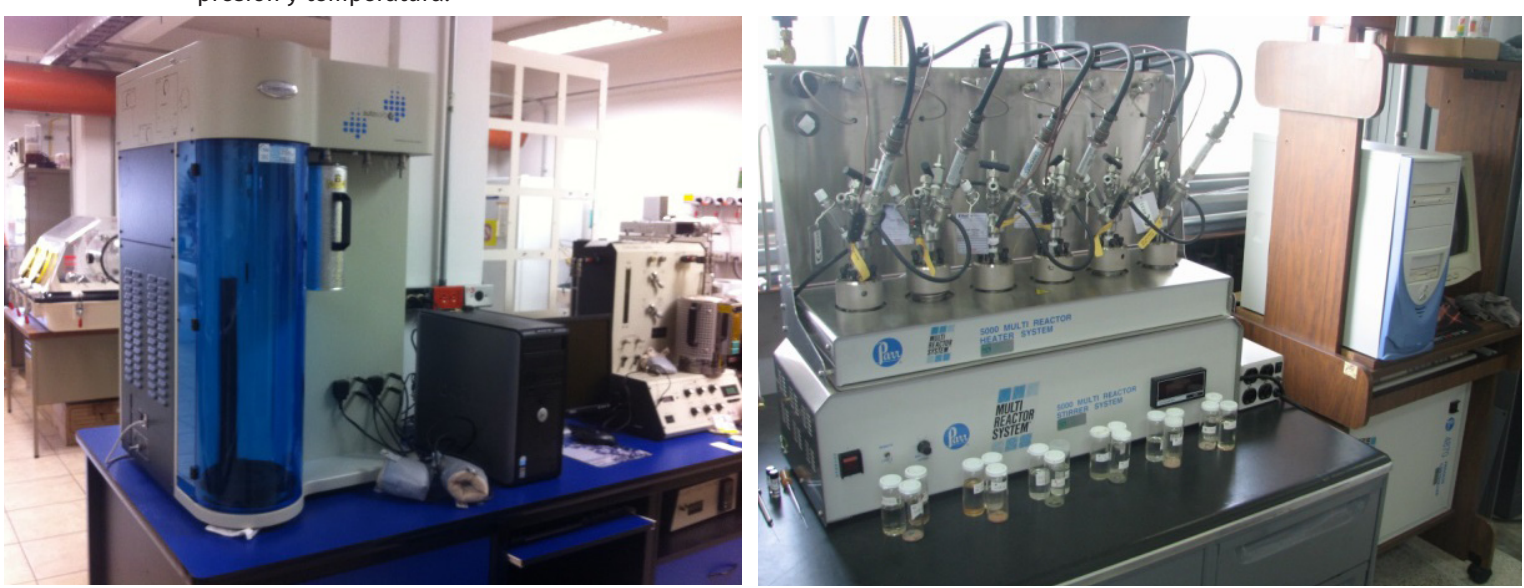
Además de la infraestructura antes mencionada, se cuenta con el siguiente equipo de uso común dentro de la UMSNH: equipo de difracción de rayos X; microscopio electrónico de transmisión; microscopio electrónico de barrido; espectrómetro de infrarrojos; absorción atómica; resonancia magnética nuclear para el hidrógeno $\left({ }^{1} \mathrm{H} \mathrm{RMN}\right)$ y equipo de microondas para preparación de materiales.

\section{Formación de recursos humanos}

El número total de estudiantes que han realizado su trabajo de grado en el área de la catálisis en nuestra institución, bajo la supervisión de los profesores que realizamos investigación en este campo es de 111 hasta la fecha. De éstos 11 fueron de doctorado, 44 de maestría y el resto de licenciatura. La gráfica siguiente muestra los porcentajes de esta distribución.

\section{Logros principales}

Son varios los logros relacionados con el área de la catálisis en nuestra Institución. La infraestructura con la que cuentan nuestros laboratorios de investigación es un éxito importante, se ha venido adquiriendo durante más de 20 años. Pero lo más sobresaliente, es la formación de jóvenes especialistas con diferentes grados académico preparados en esta área. Estos egresados se han incorporado a la vida laboral, o fomentan la investigación y educación en varas instituciones del país. En la actualidad, la catálisis es un área terminal de la maestría y de doctorado que ofrece el posgrado de la FIQ de la UMSNH, cuyos programas están acreditados en el Programa Nacional de Posgrados de Calidad del conACyT. Además de las publicaciones nacionales e internacionales en esta área de estudio, participamos frecuentemente en eventos especializados en nuestro país y en el extranjero.

FIGURA 3. Distribución de estudiantes que han terminado su grado, en problemas relacionados con la catálisis en nuestra institución.

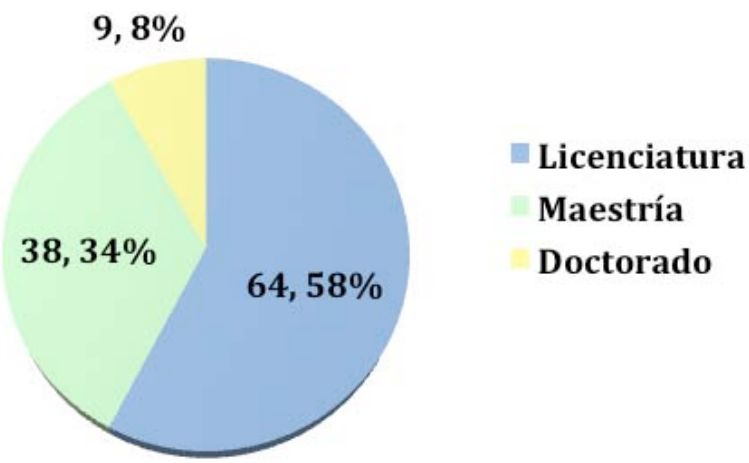

